\title{
Efeito de um Programa de Exercício Físico no Ambiente de Trabalho Sobre a Ansiedade
}

\section{EFECTO DE UN PROGRAMA DE EJERCICIO FÍSICO EN EL MEDIO AMBIENTE DE TRABAJO SOBRE LA ANSIEDAD}

Rafael Cunha Laux', Karoline Hoff², Danielle Ledur Antes ${ }^{3}$, Aline Cviatkovski ${ }^{4}$, Sara Teresinha Corazza ${ }^{5}$

1. Professor da Universidade do Oeste de Santa Catarina. Coordenador do Laboratório de Fisiologia do Exercício da UNOESC Chapecó. Chapecó - SC. Brasil.

2. Profissional de Educação Física. Seara -SC. Brasil.

3. Professora da Universidade do Oeste de Santa Catarina. Chapecó - SC. Brasil.

4. Tutora Ead da Universidade do Oeste de Santa Catarina.

5. Professora da Universidade Federal de Santa Maria. Santa Maria - RS. Brasil.

\section{RESUMO}

A pressão do mercado de trabalho somada às poucas horas de lazer têm acarretado em diversos distúrbios psicológicos, dentre eles a ansiedade, que influencia no bem estar do trabalhador, afetando até mesmo o seu rendimento profissional. Nessa perspectiva, o objetivo desse estudo foi verificar o efeito de um Programa de Exercício Físico no Ambiente de Trabalho sobre a ansiedade dos funcionários técnicos administrativos. 0 grupo de estudo foi constituído por 36 sujeitos

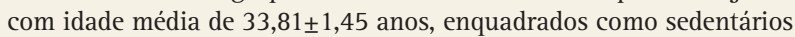
ou insuficientemente ativos pelo Questionário Internacional de Atividade Física versão VIII reduzida. A ansiedade dos sujeitos foi avaliada por meio do Inventário de Ansiedade de Beck - Bai. 0 programa de exercício físico no ambiente de trabalho foi desenvolvido utilizando exercícios de flexionamento, reforço muscular, relaxamento e alongamento. Cada aula teve duração de 10 minutos, três vezes por semana em um período de 12 semanas, totalizando 36 aulas (6 horas de atividades). Os resultados apontaram uma diminuição da ansiedade dos sujeitos na comparação entre pré e pós teste no grupo experimental $(\mathrm{p}<0,010)$, sendo que não ocorreu o mesmo no grupo controle $(\mathrm{p}=0,348)$. Conclui-se, portanto, que a intervenção com exercícios físicos no ambiente de trabalho melhorou a ansiedade dos funcionários técnico-administrativos.

Palavras-chave: SAÚDE DO TRABALHADOR, ANSIEDADE, EXERCÍCIO FÍSICO, AMBIENTE DE TRABALHO.

\section{RESUMEN}

La presión del mercado de trabajo sumada a las pocas horas de ocio ha acarreado en diversos disturbios psicológicos, entre ellos la ansiedad, que influye en el bienestar del trabajador, afectando incluso su rendimiento profesional. En esta perspectiva, el objetivo de este estudio fue verificar el efecto de un Programa de Ejercicio Físico en el Ambiente de Trabajo sobre la ansiedad de los funcionarios técnicos administrativos. El grupo de estudio fue constituido por 36 sujetos con edad media de 33,81 $\pm 1,45$ años, encuadrados como sedentarios o insuficientemente activos por el Cuestionario Internacional de Actividad Física versión VIII reducida. La ansiedad de los sujetos fue evaluada a través del Inventario de Ansiedad de Beck - Bai. El programa de ejercicio físico en el ambiente de trabajo se desarrolló utilizando ejercicios de flexión, refuerzo muscular, relajación y estiramiento. Cada clase tuvo una duración de 10 minutos, tres veces por semana en un período de 12 semanas, totalizando 36 clases (6 horas de actividades). Los resultados mostraron una disminución de la ansiedad de los sujetos en la comparación entre pre y post test en el grupo experimental ( $p<0,010)$, siendo que no ocurrió lo mismo en el grupo control $(p=0,348)$. Se concluye, por lo tanto, que la intervención con ejercicios físicos en el ambiente de trabajo mejoró la ansiedad de los funcionarios técnico-administrativos.

(Laux R, Hoff K, Ledur D, Cviatkovski A, Corazza S, 2018. Efecto de un Programa de Ejercicio Físico en el Medio Ambiente de Trabajo Sobre la Ansiedad. Cienc Trab. May-Ago; 20 [62]: 80-83).

Palabras clave: SALUD DEL TRABAJADOR, ANSIEDAD, EJERCICIO FÍSICO, AMBIENTE DE TRABAJO.

\section{INTRODUÇÃO}

Em razão das excessivas jornadas de trabalho e horas de lazer cada vez mais escassas, é possivel observar um aumento dos níveis de

Correspondencia / Correspondence

Rafael Cunha Laux

Universidade do Oeste de Santa Catarina - UNOESC,

Campus de Chapecó Av. Nereu Ramos, 3777-D - Bairro Seminário,

Chapecó-SC, Brasil CEP:898113-000.

Tel.: (049) 33192643.

e-mail: rafael.laux@unoesc.edu.br

Recibido: 08 de Febrero de 2018 / Aceptado: 08 de Junio 2018 transtornos psicológicos nos trabalhadores, dentre eles a ansiedade. Contudo, deve-se levar em consideração que a sobrecarga de trabalho pode acarretar em uma síndrome denominada Burnout, caracterizada por atingir trabalhadores que sofrem "esgotamento profissional", sendo causado pela frequente exposição a estressores emocionais e interpessoais ocupacionais. ${ }^{1}$

0 aumento dos atestados médicos provocados pelo excesso de atividades laborais têm incentivado diversas empresas em adotar programas de qualidade de vida com o intuito de promover a saúde do trabalhador por meio de exercícios físicos aplicados no próprio local de trabalho. ${ }^{2}$

Estimativas revelam que 1,1 milhão de indivíduos morrem por causa de lesões e doenças relacionadas ao trabalho, mas felizmente está crescendo o número de empreendedores que investem em Programas de Promoção da Saúde do Trabalhador. Com os 
Programas de Promoção da Saúde do Trabalhador, tanto as empresas como seus funcionários são beneficiados, o empregador ganhará um funcionário mais saudável e, por consequência, mais produtivo, além de um ambiente eficiente e seguro, por sua vez, o empregado terá a saúde otimizada e maior disposição para desempenhar sua função. ${ }^{3}$

Os Programas de Exercícios Físicos no Ambiente de Trabalho (PEFAT), são conhecidos no Brasil como Ginástica Laboral. ${ }^{4}$ Esses programas consistem desde simples intervenções com alongamento, quanto em programas mais complexos com um conjunto de exercícios físicos específicos para compensar os malefícios do trabalho, no qual são realizados atividades lúdicas e exercícios para reforço muscular e para melhora ou manutenção dos índices de flexibilidade. ${ }^{5}$

Existem pesquisas que comprovam os benefícios à saúde dos praticantes dos PEFAT, sejam eles nos modelos tradicionais, como a melhoria na percepção de dores e absenteísmo ${ }^{6}$, menor prevalência de inatividade física e diminuição no consumo abusivo de álcool ${ }^{7}$, melhora da motricidade fina ${ }^{2}$, redução de algias osteomusculares na coluna vertebral e alguns segmentos corporais ${ }^{8}$, diminuição da intensidade e frequência da dor muscular e melhora da postura sentada. ${ }^{9}$ Com a estrutura do programa de exercício físico no ambiente de trabalho existe estudo que destacam a diminuição dos atestados médicos ${ }^{5}$, melhora no tempo de reação simples e de escolha, benefícios para o estado de humor dos funcionários. ${ }^{10}$

Os benefícios apontados pelos PEFAT se mostram importantes, pois o trabalho é uma atividade diária que ocupa grande parte do tempo do indivíduo e, portanto, diminui o tempo de ócio ou de lazer do sujeito, assim influenciando em sua qualidade de vida, causando aumento do cansaço mental, perda de memória imediata, dificuldade de atenção e concentração, sentimento de insatisfação, crises de ansiedade e humor depressivo, afetando até mesmo o seu rendimento profissional. ${ }^{11}$

Nessa perspectiva, o objetivo deste estudo foi verificar o efeito de um Programa de Exercícios Físicos no Ambiente de Trabalho sobre a ansiedade dos funcionários técnicos administrativos da Universidade Federal da Fronteira Sul - Chapecó.

\section{MATERIAL E MÉTODOS}

Essa pesquisa caracterizou-se como experimental. ${ }^{12}$ Após trinta e seis aulas, durante um período de doze semanas, três vezes por semana do PEFAT realizou-se o teste de ansiedade. 0 projeto foi aprovado pelo Comitê de Ética em Pesquisa (CEP) da Universidade Federal da Fronteira do Sul CAAE n ${ }^{\circ}$ 53085216.5.3001.5564.

A população do estudo foi formada por 75 servidores técnicos administrativos da Universidade Federal da Fronteira Sul Chapecó. 0 grupo de estudo foi constituído por 36 servidores técnicos dos setores administrativos entre 18 e 50 anos de idade, enquadrados como sedentários ou insuficientemente ativos pelos critérios do Questionário Internacional de Atividade Física (IPAQ) versão VIII reduzida. 0 grupo foi selecionado conforme os seguintes preceitos: a realização de um convite, bem como divulgação da UFFS, anamnese aplicada com o intuito de identificar a idade e se pratica atividade física regularmente, a definição dos participantes. A divisão dos grupos foi de forma igual por meio de um sorteio simples, sendo 18 integrantes no Grupo Controle (GC) e no Grupo Experimental (GE), conforme descrito na figura 1.
Figura 1.

Desenho experimental do estudo.

\begin{tabular}{|l|l|l|l|}
\hline $\begin{array}{l}\text { Seleção do Grupo } \\
\text { de Estudo }\end{array}$ & Pré teste & Grupo Controle 1 & Re-teste \\
& Pré teste & Grupo Experimental 1 & Re-teste \\
\hline
\end{tabular}

Foram excluídos os sujeitos que fizeram uso de algum medicamento que poderia trazer efeito para o sistema nervoso central, que demonstraram mudanças nos níveis de prática de atividade física durante o estudo e também os que não apresentaram 75\% de frequência mínima.

Os instrumentos utilizados para a coleta de dados foram: a) Anamnese para a caracterização dos sujeitos, bem como uma avaliação das medidas antropométricas de massa corporal e estatura, e a partir disso foi calculado o Índice de Massa Corporal $\left(\mathrm{IMC}=\right.$ Massa Corporal /(Estatura $\left.{ }^{2}\right)$, sendo que a massa foi expressa em quilogramas (Kg) e a estatura em metros (m); b) Questionário Internacional de Atividade Física (IPAQ) versão VIII reduzida, para calcular o nível de atividade física, de acordo com a classificação: a) Sedentário, quando não realiza nenhuma atividade física por pelo menos 10 minutos contínuos na semana; b) Insuficientemente Ativo que subdivide-se em: Insuficientemente Ativo A, realiza atividade física por pelo menos 10 minutos contínuos 5 dias/ semana ou 150 minutos/semana; e Insuficientemente Ativo B, que não segue os critérios de recomendação citada nos indivíduos insuficientemente ativos A e pratica pelo menos 10 minutos de atividade física contínua; 13 c) Inventário de Ansiedade de Beck - Bai para mensurar o nível de ansiedade dos participantes, respeitando a seguinte classificação: 0 a 10 - dentro do limite mínimo (ansiedade mínina); 11 a 19 - ansiedade leve; 20 a 30 - ansiedade moderada; 31 a 63 - ansiedade grave. ${ }^{14,15}$

0 programa de exercício físico no ambiente de trabalho foi desenvolvido utilizando a proposta de PEFAT de Laux et al. ${ }^{4}$ Cada aula teve duração de 10 minutos, três vezes por semana em um período de 12 semanas concluindo 36 aulas (6 horas de atividades), utilizando exercícios de flexionamento, reforço muscular, relaxamento e alongamento.

A intervenção foi composta por parte inicial, principal e final. A parte inicial teve duração de quatro minutos, com destaque para as atividades recreativas, envolvendo ritmo, coordenação motora fina, rapidez de raciocínio e atenção, utilizando-se de bolinhas de borracha, jornal, bola de vôlei, arcos, bastões, músicas. Na parte principal foram desenvolvidos os exercícios de flexionamento com duração de cinco minutos ou os exercícios de reforço muscular com duração de quatro minutos, com ênfase nos grupos musculares mais utilizados durante o trabalho. Os materiais utilizados foram bolinhas de borrachas, bastões, arcos, colchonetes, músicas. Já, na parte final foram desenvolvidos os exercícios de flexionamento voltados para atividades de relaxamento com duração de um minuto e exercícios de reforço muscular com alongamentos, os mesmos das sessões de flexionamento, com intensidade submáxima e duração de dois minutos, envolvendo exercícios de concentração e respiração, dinâmicas de grupo e alongamento, com o uso de colchonetes e músicas (figura 2). ${ }^{4,5}$

0 controle da intensidade dos exercícios foi realizado pela escala de percepção subjetiva do esforço de Borg16 (2000), de modo a controlar a intensidade durante toda atividade entre pouco intensa e intensa. 
Figura 2.

Estrutura das sessões do PEFAT.

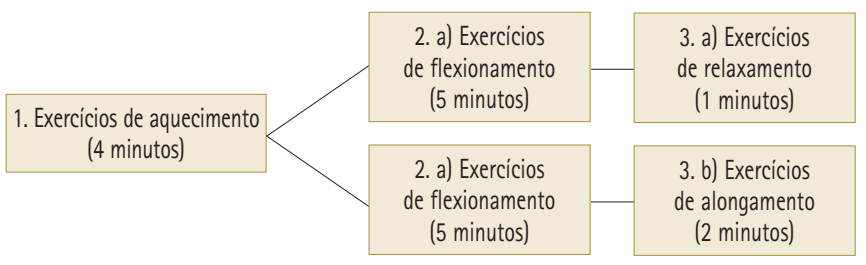

Fonte: Adaptado de Laux et al. (2018).

Para a análise dos dados foi utilizado estatística descritiva e inferencial. Para as comparações utilizou-se o teste de Wilcoxon entre os períodos (pré e pós) e o teste Mann Whitney e o teste T para amostras independentes entre os grupos. Na comparação entre os níveis de atividade física utilizou-se o teste Exato de Fisher. Os testes foram realizados no programa estatístico SPSS ${ }^{\circledR}$ versão 21.0 para Windows, com nível de significância de 5\%.

\section{RESULTADOS}

Participaram do estudo 36 sujeitos classificados como sedentários

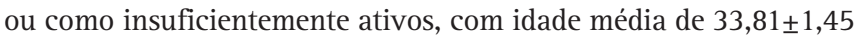
anos, subdivididos em Grupo Controle e Grupo Experimental. Na Tabela 1 são apresentados os dados de caracterização do grupo. Percebe-se a ausência de diferença estatisticamente significativa em todas variáveis apresentadas.

Tabela 1.

Caracterização do grupo.

\begin{tabular}{|c|c|c|c|c|c|c|}
\hline & \multicolumn{2}{|c|}{$\begin{array}{c}\text { Grupo Controle } \\
\qquad(n=18)\end{array}$} & \multicolumn{4}{|c|}{$\begin{array}{l}\text { Grupo Experimental } \\
(n=18)\end{array}$} \\
\hline & Média & DP & & Média & DP & $P$ \\
\hline Idade (anos) & 32,78 & 6,67 & & 34,83 & 10,49 & $0,92 b$ \\
\hline Massa Corporal (Kg) & ) 76,51 & 20,01 & & 74,47 & 16,31 & $0,74 a$ \\
\hline Estatura (m) & 1,68 & 0,10 & & 1,69 & 0,08 & $0,79 a$ \\
\hline IMC (Kg/m²) & 27,06 & 6,20 & & 25,96 & 4,47 & $0,54 a$ \\
\hline IPAO & $\begin{array}{l}\text { Pré } \\
\mathrm{n}(\%)\end{array}$ & $\begin{array}{l}\text { Pós } \\
n(\%)\end{array}$ & $P$ & $\begin{array}{l}\text { Pré } \\
\mathrm{n}(\%)\end{array}$ & $\begin{array}{l}\text { Pós } \\
\mathrm{n}(\%)\end{array}$ & $P$ \\
\hline Sedentário & $4(11,11 \%)$ & $1(2,77 \%)$ & & $7(19,44 \%)$ & $6(16,66 \%)$ & \\
\hline & & & $0,34 c$ & & & $1,00 \mathrm{c}$ \\
\hline Insufi. Ativo & $14(38,88 \%)$ & $17(47,22 \%)$ & & $11(30,55 \%)$ & $12(33,33 \%)$ & \\
\hline
\end{tabular}

${ }^{*} p<0,05$; a teste T não pareado; $b$ Mann Whitney test; $c$ teste exato de Fisher.

Na Tabela 2 são apresentados os valores da ansiedade, nos quais percebeu-se uma diminuição ao comparar o pré e pós teste no grupo experimental $(\mathrm{p}<0,010)$, sendo que não ocorreu o mesmo no grupo controle $(p=0,348)$. Na comparação entre os pós testes nos grupos verificou-se também uma diferença entre o grupo controle e o grupo experimental $(\mathrm{p}<0,049)$.

Tabela 2.

Ansiedade.

\begin{tabular}{|c|c|c|c|c|c|}
\hline & Grupo controle & & Grupo experiment & & $p$ entre \\
\hline & Mediana (25-75) & $p$ & Mediana (25-75) & $p$ & grupo \\
\hline & Pré 9,00 $(6,00-12,50)$ & & $8,50(1,75-18,00)$ & & 0,800 \\
\hline $\begin{array}{l}\text { Ansiedade } \\
\text { (pontos) }\end{array}$ & Pós 9,50 $(4,00-17,50)$ & 0,348 & $4,00(1,65-10,00)$ & $0,010^{*}$ & $0,049 \#$ \\
\hline
\end{tabular}

${ }^{*} p<0,05$ no teste de Wilcoxon; $\# p<0,05$ no teste Mann Whitney.

\section{DISCUSSÃO}

Ao verificar os efeitos de um PEFAT de curta duração sobre a ansiedade de técnicos administrativos, observou-se uma melhora na variável estudada ao realizar a comparação entre pré e pós teste no grupo experimental, ressaltando que essa mudança não ocorreu no grupo controle.

Os resultados dessa pesquisa diferem-se do estudo realizado por Freitas et $\mathrm{al}^{17}$,no qual avaliaram-se os efeitos de um programa de atividade física sobre os níveis de ansiedade de uma equipe de enfermagem de cuidados paliativos, com sessões de cinco vezes por semana, com duração de 10 minutos durante três meses, porém não obtiveram diminuição nos níveis de ansiedade dos sujeitos. Os exercícios não foram controlados quanto a sua intensidade, além de não possuir um grupo controle, dificultando a análise mais aprofundada dos dados.

A eficácia do exercício físico sobre a ansiedade pode ser encontrada na revisão realizada por Chu et $\mathrm{al}^{18}$, o qual contemplou estudos publicados entre 1990 e 2013, que realizaram intervenções na área da dança, exercício aeróbio, treinamento resistido e exercícios de ioga e concluíram que essas atividades realizadas no ambiente de trabalho diminuíram os sintomas da ansiedade. Essa relação entre exercício físico e ansiedade é evidenciada no estudo de Khanzada et $\mathrm{al}^{19}$, o qual demonstra que os maiores níveis de ansiedade se encontram naqueles sujeitos que não praticam atividade física regularmente.

Destaca-se, entre os achados do presente estudo, a melhora do grup o experimental comparado com o grupo controle após a intervenção, destacando a eficácia do programa e que o mesmo conseguiu diminuir para menos da metade os sintomas de ansiedade, enquanto no grupo controle esses índices aumentaram.

Existem poucos estudos do tipo experimental e que trazem resultados sobre o efeito de um PEFAT com sessão diminuída (10 minutos de intervenção) sobre a ansiedade. Porém, na área da psicologia do esporte, existem algumas pesquisas que comprovam o efeito benéfico do exercício físico sobre a ansiedade, como o estudo de Herring et $\mathrm{al}^{20}$, no qual foi proposto um programa de treinamento de exercícios aeróbios e resistidos com duração, em média, de três sessões por semana de 30 minutos em um período não superior a três meses, resultando na redução dos sintomas da ansiedade crônica, destacando ser de grande utilidade para pacientes que optam por tratamentos não-farmacológicos. Em um estudo realizado por Sardinha et $\mathrm{al}^{21}$, no qual foi aplicado um programa de exercício supervisionado, incluindo aeróbio, alongamento e treinamento de força em 42 coronariopatas portadores de transtornos psiquiátricos, com sessões de 20 a 30 minutos, que concluiu que praticar exercício físico regularmente diminui a probabilidade de desenvolver transtornos mentais.

0 PEFAT com sessão diminuída (10 minutos de intervenção) demostrou um efeito positivo sobre a ansiedade, assim como as sessões de 20 a 30 minutos recomendadas por vários estudiosos, o que é ideal para aplicação no ambiente de trabalho, pela economia de tempo total na intervenção, diminuindo o custo efetivo do programa para o empregador. ${ }^{22}$

Além da economia no tempo de execução do PEFAT, a implantação desse tipo de intervenção não farmacológica é importante no ambiente de trabalho, pois segundo Muller $^{23}$ existe uma alta prevalência e incidência de transtornos mentais e comportamentais relacionados ao trabalho, ocupando a segunda e a terceira posição no Brasil como os principais responsáveis pelas causas de afastamentos 
médicos. De modo que o trabalho ocupa grande parte do tempo do indivíduo, tornando-se um excelente local para investimentos em saúde, sendo importante ressaltar os inúmeros motivos para aderir programas de exercício físico, como redução nos cuidados de saúde, diminuição dos custos e atestados médicos, resultando, consequentemente, em maior produção. ${ }^{24}$

Conclui-se, diante das evidências relatadas, que houve uma melhora na ansiedade dos sujeitos que participaram do programa de exercício físico de curta duração no ambiente de trabalho, mostrando o quão eficaz essa intervenção é na diminuição dos níveis de ansiedade, sendo mais uma ferramenta para a saúde psicológica do trabalhador. Algumas limitações são destacadas ao término desta investigação, como o recente advento deste tema, os estudos, principalmente do tipo experimental, encontram-se ainda escassos na literatura científica. Outras limitações também ocorreram, como a pequena quantidade de sujeitos que participaram da intervenção, além do tempo limitado de execução do programa. Porém, a exigente seleção dos participantes e o controle de variáveis intervenientes (nivel de prática de atividade física, frequência e intensidade da intervenção) fortalecem os achados da presente pesquisa.

\section{REFERÊNCIAS}

1. Hyeda A, Handar Z. Avaliação da produtividade na sindrome de Burnout. Rev Bras Med Trab. 2011; 9(2):78-84.

2. Mezzomo SP, Ribeiro A, Corazza ST. Os efeitos da ginástica laboral sobre as habilidades básicas de funcionários de setores administrativos. R Bras Cien Saúde. 2010; 8(25):6-13.

3. Martins CO, Michels G. Programas de promoção da saúde do trabalhador: exemplos de sucesso. Rev bras cineantropom desempenho hum. 2003; 5(1):85-90.

4. Laux RC, Corazza ST, Andrade A. Programa de Exercício Físico no Ambiente de Trabalho: Uma proposta de intervenção. Rev Bras Med Esporte. 2018; 24(3):238-242.

5. Laux RC, Pagliari P, Effting JV, Corazza ST. Programa de Ginástica Laboral e a Redução de Atestados Médicos. Cienc trab. 2016; 18(56):130-133

6. Gomes HR Vieira LL, Santos RG, Soares PM. Percepção de praticantes de ginástica laboral sobre parâmetros de saúde geral e relacionada ao trabalho. Coleç Pesqui Educ Fis. 2012; 11(5):113-120

7. Grande $\mathrm{AJ}$ et al. Comportamentos relacionados à saúde entre participantes e não participantes da ginástica laboral. Rev Bras Cineantropom Desempenho Hum. 2011;13(2):131-137.

8. Freitas FCT. A Ginástica laboral na redução de queixas de estresse ocupacional e dor osteomuscular em funcionários administrativos de uma Universidade pública. [dissertação]. São Paulo: Universidade de São Paulo; 2010. 169 p.

9. Candotti CT, Stroschein R, Noll M. Efeitos da ginástica laboral na dor nas costas e nos hábitos posturais adotados no ambiente de trabalho. Rev Bras Ciênc Esporte. 2011; 33(3):699-714.

10. Laux RC. Efeitos da intervenção com exercícios físicos no ambiente de trabalho sobre o tempo de reação e o humor [dissertação]. Santa Maria-RS: UFSM; 2016. 78 p.

11. Lipp MEN. Transtorno de adaptação. Bol-Acad Paul Psicol. 2007; 27(1):72-82.

12. Thomas JR, Nelson JK. Métodos de Pesquisa em Atividade Física. $6^{a}$ ed. Porto Alegre: Artmed; 2012.

13. Souza Vespasiano B, Dias R, Alves D. A utilização do Questionário Internacional de Atividade Física (IPAO) como ferramenta diagnóstica do nivel de aptidão física: uma revisão no Brasil. Saúde Rev. 2012; 12(32):49-54.

14. Cunha JA. Inventário Beck de Ansiedade (BAl); Manual da versão em português das escalas Beck. São Paulo: Casa do Psicólogo; 2001.

15. Terra FS. Avaliação da ansiedade, depressão e autoestima em docentes de Enfermagem de universidades pública e privada [dissertação]. Ribeirão Preto: Universidade de São Paulo; 2010. 260 p.

16. Borg G. Escalas de Borg para a dor e o esforço percebido. Sao Paulo: Manole; 2000.

17. Freitas AR, Carneseca EC, Paiva CE, Ribeiro BS. Impacto de um programa de atividade física sobre a ansiedade, depressão, estresse ocupacional e síndrome de Burnout dos profissionais de enfermagem no trabalho. Rev Latino-Am Enfermagem. 2014; 22(2):332-336.

18. Chu AH, Koh D, Moy FM, Müller-Riemenschneider F. Do workplace physical activity interventions improve mental health outcomes?. Occup Med. 2014; 64(4):235-245.

19. Khanzada FJ, Soomro N, Khan SZ. Association of Physical Exercise on Anxiety and Depression Amongst Adults. J Coll Physicians Surg Pak. 2015; 25(7):546-548.

20. Herring MP, O'Connor PJ, Dishman RK. The effect of exercise training on anxiety symptoms among patients: a systematic review. Arch Intern Med. 2010; 170(4):321-331.

21. Sardinha A, Gil C, Cardoso A, Nardi AE. Prevalência de transtornos psiquiátricos e ansiedade relacionada à saúde em coronariopatas. Rev Psiq Clin. 2011; 38(2):61-65.

22. Laux RC. Workplace Physical Activity Intervention Programs: Costs and Benefits. Inter Phys Med Rehab J. 2017;1(4):107-108.

23. Müller FG. Perícia psicológica de transtornos mentais relacionados ao trabalho [dissertação]. Florianópolis: Universidade Federal de Santa Catarina; 2014. 235 p.

24. Baicker $K$, Cutler $D$, Song Z. Workplace wellness programs can generate savings. Health Affair. 2010; 29(2):304-311. 